\title{
CATALOGUE OF THE FIRST NATIONAL LOAN EXHIBITION NATIONAL ART ASSOCIATION
}

N

6510

C 38

1892

NMAA

-3k

OPEN FROM WEDNESDAY, THE EIGHTEENTH OF MAY, TO FRIDAY, THE TWENTY-SEVENTH OF MAY, INCLUSIVE. HOURS OF EXHIBITION FROM NINE-THIRTY A.M. TO FOUR-THIRTY P. M.

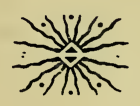

IN THE CHAPEL OF THE SMITHSONIAN INSTITUTION, WASHINGTON, D. C., 1892. 


\title{
NATIONAL ART ASSOCIATION MDCCCXCII.
}

\section{㴘}

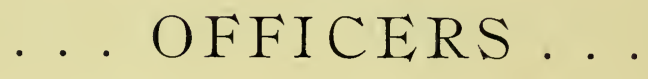

HONORARY PRESIDENT.

MRS. HARRISON.

HONORARY VICE-PRESIDENTS.

VICE-PRESIDENT AND MRS. MORTON.

\author{
PRESIDENT. \\ DANIEL HUNTINGTON. \\ VICE-PRESIDENTS.
}

Albert Bierstadt,

F. D. Millet,

Augustus St. Gaudens,

William M. Chase,

Richard M Hunt,

Stanford White,

Edmund Clarence Stedman,

John Armstrong Chanler, “

Mrs. Candace Wheeler,

Mrs. S. V. R. Cruger,

Charles L. Hutchinson, Chicago. James W. Ellsworth,

TREASURER.

J W. THOMPSON
New York. Mrs Potter Palmer,

Chicago. Mrs. George Hearst, San Francisco. Mrs. Bellamy Storer, Cincinnati. Mrs. H. K. Porter, Pittsburgh. E. Francis Riggs, Washington.

C. M. FFOULKE, JEFFERSON CHANDLER, Thomas E. Waggaman, Beriah Wilkins, Charles J. Singer, Francis Colton, M. M. PARKER,

SECRETARY. KATE FIELD. 
$N$

6510

C38

1892

NMAA

NATIONAL ART ASSOCIATION

棌

\section{AR'T COMMITTEES \\ NEW YORK.}

Albert Bierstadt, $\quad$ F. D. Millet, H. W. Watrous,

H. Bolton Jones, C. Y. Turner.

F. P. Vinton, $\begin{aligned} & \text { BOSTON. } \\ & \text { J. Foxcroft Cole.* }\end{aligned}$

CHICAGO.

W. M. R. French, John Vanderpoel, C. D. Grover.

PHILADELPHIA.

Thomas Eakins, Clifford Grayson, Charles Linford.

WASHINGTON.

E. F. Andrews, C. H. L. Macdonald,

H. Hobart Nichols,

* Since deceased.

NMAP. M. SAWERJ

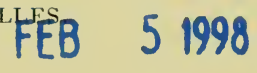




\section{WING to the generous subscriptions of the following patriotic}

men and women, who realize that the advancement of art means the elevation of the people, the propaganda in behalf of free art and the object-lesson of a National Loan Exhibit have been made possible.

Mrs. George Hearst, Mrs Potter Palmer, Mrs. H. K. Porter, Mrs J H. Walker, Mrs. Thomas Lowry, Mrs Candace Wheeler, Mrs Dora Wheeler Keith, Mrs. Albert Cliffford Barney, Mrs. Anna lea Merritt, Mrs. M H. Wilmarth, Mrs. Mary Stuart Armstrong, Mrs. Chakles T. Yerkes, Mrs M. H. De Young, C. R. S., Mrs. N. S. Chapin, Mrs. C. C. Buel, Mrs. Frank Baker, Miss Lilian Whiting, Mrs. CoOper, Miss Nichols, Mrs. McCarthy, Mrs. Evans, Washington Society of Artists, Thomas E. Waggaman, Beriah WILKINS, Charles J. Singer, E. FraNCIS RigGS, J W. Thompson.
C. M. FFoulKe,

Gardiner G. Hubbard, Francis Colton, SaMuel G Ward, T. E Roessle, Edmund Clarence Stedian. Albert Bierstadt, ЈACOB SCHIFF, J. J. HAGERMAN, Marshall Field, Charles L. Hutchinson, Martin A. Ryerson, George M. Pullman, James W. Ellsworth, James H. Dole, Michael Cudahy. William Schaus, S P. Avery, Daniel Huntington, R. W. Gilder,

S. B. C., Louis Prang, George H. Boughton, Donald Fletcher, Sanuel Elliott,

C. S. Elguth, G J. GrLbert, J. M. MCKeown. 


\section{CATALOGUE. \\ 然}

Artist.

TitLE.

OWNER.

I. Iron Workers.

Thomas Anschutz.

2 Afternoon on the River.

W. S. Allen.

3 Spring.

E. A. Abbey.

Thomas B. Clark.

Artist.

F. D. Millet.

4 Fisherman's Daughter.

Henry Bacon.

5 Hester Prynne.

Geo. H. Boughton.

6 Watching the Train.

J. G. Brown.

$$
7 \text { Portrait. }
$$

Cecilia Beaux.

8 Music.

E. H. Blashfield.

Francis Colton.

Stilson Hutchins.

Thomas B. Clark.

Sabin W. Colton.

Thomas B. Clark.

9 The Canoe Builder.

Ralph Albert Blakelock.

Mrs. H. W. Watrous. Io Bothered.

George H. Butler. Thomas B. Clark.

Howard Russell Butler.

I 1 Marine.

I 2 In the Yellow Light.

J. Carroll Beckivith. Artist.

Dennis Bunker. I 3 Landscape.

Artist. Artist. 
ARTIST.

Albert Bierstadt.

William A. Coffin.

i6 The Mediterranean Sea.

F. E. Church.

C. 'T. Chapman.

TITLE.

OWNER

I 4 Lak̉e Louise.

15 Sunset.
Artist.

Artist.

Thomas B. Clark.
Artist.

I 8 The Pursuit of the Ideal.

Kenyon Cox.

Artist.

I9 Moonlight Street-Scene, Italian.

C. C. Coleman.

Artist.

20 Mother and Child.

William M. Chase.

2 I Evening.

Henry Dearth.

Thomas B. Clark.

22 Hung on the Line.

Joseph Decker.

E. Daingerfield

23 After Milking.

24 Christ and the Fisherman.

Frank Du Mond.

Thomas B. Clark.

T. H. Dewing.

Ruger DoNoho.

W. H. Dannat.

WyatT Eaton.

25 Portrait.

26 Wood Interior.

27 Après la Messe.

Artist.

Artist.

Stanford White.

Artist.

Charles J. Singer.

28 Sappho.

Thomas B. Clark. 
Artist.

Title.

OWNKR.

Thomas Eakins.

29 Cowboys At the Home Ranch.

George Fuller.

30 Quadroon.

$3^{\text {I }}$ Wounded to the Rear.

Gilbert Gaul.

EDward Gay.

$$
3^{2} \text { In May. }
$$

Artist.

Mrs. S. D. ITarren.

Thomas B. Clark.

Frank R. Green.

33 Sweetheart.

S. J. Guy,

34 Out of His Element.

Clifford Grayson.

35 Portrait.

$3^{6}$ Sunbeams.

Hamilton Hamilton.

Thomas Hovenden.

37 Chloe and Sam.

$3^{8}$ Goldsmith's Daughter.

D. Huntington.

39 Portrait of Longfellow.

(Painted in 1863 for Ticknor \& Fields.)

G. P. A. Healy. 40 Moonlight.

Alexander Harrison.

WILLIAM HART.

4I A Summer Idyll.

WilliaM M. Hunt

42 Girl With the Cows.

John Armstrong Chanler.

43 After a Shower on the Delaware River.

George InNess.

James $\mathrm{IV}$. Ellsworth. 
Francis C. Jones. 44 The Favorite Grandchild.

Eastman Johnson.

$$
45 \text { Eastman Johnson. }
$$

46 View of Ulleswater.

J. F. Kensett.

47 Early Spring.

Ridgway Knight.

48 Head of Child.

Mrs. Dora Wheeler Keith.

Artist.

Artist.

Miss Riggs.

C. J. Singer.

Artist.

49 Landscape.

Charles Linford.

Charles Lasar.

50 The Widow.

Artist.

John Armstrong Chanler.

5 I The Great Statue of Armida Buddha at Namakura, Japan, known as the Daibutsu, from the Priest's Gardẹn. (Water-color.)

JoHN LA FARGE.

Artist.

$5^{2}$ Death of the Hamadryad.

Frank Moss.

53 Ideal Head.

Stanley Middleton.

Artist.

Francis Miller.

54 Local Freight Caboose.

55 Portrait of F. D. Millet.

George W. Maynard.

Thomas B. Clark.

Artist.

F. D. Millet.

$5^{6}$ Portrait.

Anna Lea Merritt.

Mrs. George Carpenter.

57 At the Inn.

F. D. Millet.

Union League Club, New York. 
Artist.

TITLE.

OWNER.

$5^{8}$ Diana.

Frederick William McMonnies.

Stanford White.

J. MCEntee.

59 Autumn.

J. C. Nicoll.

60 Sunlight on the Sea.

6r Mother and Child.

E. Wood Perry.

Miss Riggs.

Artist.

Artist.

62 Spring Morning.

Arthur Parton.

63 September Sunshine.

William L. Picknell.

64 A Red Flower.

ROBERT REID.

$$
6_{5} \text { Dryads. }
$$

Albert H. Ryder.

Stanford IThite.

66 Street in Barbizon-Sunset Glow.

TheOdore Robinson.

John Armstrong Chanler.

Roswell D. Sawyer. 67 Daphne.

George H. Smillie.

Thomas B. Clark.

69 Bronze Medallion-Head of the Gilder Boy.

Augustus St. Gaudens.

Stanford White.

JOHN S. SARGENT.

70 Venice.

William T. Trego.

7 I Battery Forward.

James G. Tyler.

72 First American Shipwreck.

Thomas B. Clark. Stanford White. 
ARTIST.

Title.

OWNer.

C. Y. Turner.

73 John Alden's Letter.

Abbott H. Thayer,

74 Portrait.

J. H. Twachtman.

75 Winter.

Artist.

Stanford White.

John Armstrong Chanler.

76 Accused of Witchcraft.

Douglas Volk.

Thomas B. Clark.

77 A Landscape.

R. W. VAN Boskerck.

78 Marsyas.

Elihu Vedder.

$$
78 \text { Marsyas. }
$$

79 Gen. Devens.

F. P. Vinton.

Department of Justice.

J. H. WitT.

8o Good Bye.

8I The Silver Wedding.

H. W. WATrous.

82 Home By the Sea.

WV. Whittredge.

Thomas B. Clark.

\section{ADDENDA.}

Artist.

Title.

OWNer.

83 Portrait of Mrs. S.

LeA Robbins.

84 North Conway.

A. F. Bellows.

$8_{5}$ The Wedding Morning.

Henry Mosler.

C. J. Singer.

Stilson Hutchins.

Stilson Hutchins. 
ARTist.

J. B. BRistol.

JOSEPH JEFFERSON.

Thomas Moran.

89 The Provincial Asylum.

WaLter Gay.

Charles H. Davis.

W. H. Low. 90 Winter Eve.

9I Love Disarmed.
OWNER.

86 On The Connecticut.

87 Louisiana Swamp.

88 Knabe Canyon.

Stilson Hutchins.

Stilson Hutchins. Major J. W. Powell.

Thomas E. Waggaman.

Thomas E. IVaggaman.

Gardiner G. Hubbard.

92 Bust of Winslow Homer.

Wy. Rudolph O'Donovan.

Thomas Eakins.

93 The Return.

E. E. Simmons.

Artist.

94 An Evening Effect.

Mrs. George Hearst.

Carl Marr.

95 The First Communion.

R. N. VONNOH.

$9^{6}$ Portrait of Little Phobe.

Mrs. George Hearst.

O? $?^{\text {RRIN PECK. }}$

Mrs. George Hearst. 97 Love's Token.

Mrs. George Hearst. 
SMITHSONIAN INSTITUTION LIBRARIES

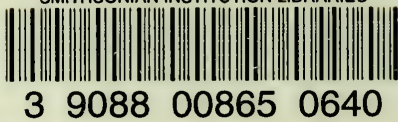

